\title{
TIME SCALES FOR THEORY AND PRACTICE
}

\author{
Gernot M. R. Winkler \\ U.S. Naval Observatory \\ Washington, DC 20392-5100
}

Report given to the XXI General Assembly of the IAU

July 1991

\section{INTRODUCTION}

Very early human experience has suggested a practical definition for the measurement of time: We define a unit of time by defining a standard (cy ilical) process. Whenever this process completes its cycle identically, a unit of time has elapsed. This is the origin for the various measures of time in classical astronomy. Nature suggests strongly that we use as such standard processes the year (defined as a complete revolution of the earth around the Sun), the month (the completion of a revolution of the moon around the earth), and the day which again can be measured in several different ways. While the sidereal day is measured by a rotation in respect to the vernal equinox, the mean solar day is measured in respect to the mean. Sun. More recently, we have distinguished many more different ways of defining measures of time, partly in response to perceived needs of the applications, but in part also from purely aesthetic principles.

Nevertheless, behind all these possible different choices for measuring time, there is the ideal concept which we must keep in mind when we deal with the details. Newton's approach has been to assume time to flow uniformly and equally everywhere but we have known since 1905 that this is not a sufficient basis for scientific applications.

Until 1955, the unit of time had been the Second as a subunit of a mean solar day. Later we tried to overcome the known variability of the speed of rotation of the earth by using its orbital motion around the Sun as the time standard. This produced the old "Ephemeris Second" as the standard.

Since 1967, we use as the standard the duration of $9,192,631,770$ cycles of a microwave resonance frequency of the Cesium atom under specified conditions. The new definition has been set to agree, within the errors of measurement, with the traditional measure of time which is dictated by our dependency on the Sun. This new standard, however, has the great advantage that it can be easily realized anywhere; i.e., we can carry our time measure with us wherever we go. In using it we must, however, pay attention to the necessity of making certain transformations of our measures of events in systems which are in motion relative to us. This necessity arises precisely because of the purely relational (as a relation between 
processes) nature of our time concept. The mathematical relations constitute what is known as relativity theory.

\section{RELATIVITY}

The theory is based on the striking and paradoxical experimental evidence that the most fundamental connection between events, energy travelling through free space, i.e., electromagnetic radiation such as light, travels at finite speed and that this speed is the same for all observers regardless of their state of motion. The fact that this finding is paradoxical must alert us to the danger of bringing "innate" ideas to our scientific thinking about the world. But this is only the first instance for the sobering realization that nature has no obligation to conform with our pre-conceived notions. Or, expressed differently, that we cannot expect to always succeed when we extend the experience and ideas from our everyday world into new domains of scientific exploration.

Relativity theory (the special theory which can be considered as thoroughly established and tested) leads to these conclusions:

a. Succession of causally relatable events is invariant (absolute), i.e., their order is fixed.

b. Succession of causally unrelated events is arbitrary; it depends on the reference system chosen. The order of such events can be inverted in a different reference system.

c. Only causally unrelated events can be made simultaneous by selecting an appropriate reference frame.

d. Time as such, i.e., as parameter of change, has a local meaning which can be unambiguously extended to remote locations only to the extent as causal connections exist or can be established by means of light signals.

e. Time is not a coordinate but a parameter which evolves unidirectionally commensurate with change. As a purely mathematical device, it can be represented as a fourth coordinate by keeping in mind its principally different character as parameter of change which does not allow arbitrary transiations as space coordinates do. In particular, the separation of the future from the past is absolutely fixed according to (a.) above, for a given location itself. It is not absolutely objective for the distant areas in space.

f. Since there is no unambiguous common time measure between causally unrelated regions in space, space itself (also to be conceived as purely relational) is not a "thing". In the sense of a frame of co-existence it has a dynamic, i.e., time frame dependent character.

g. Time and causality (which has as a necessary and sufficient condition some energy transfer which takes time) are therefore more fundamental concepts than space in the sense that time is not space-like but space is time dependent.

Despite (f.), there could be a quasi cosmic or universal time but only if all world lines have a common intersection, i.e. in the case of a big bang origin. On the basis of our present concepts such a natural cosmic time seems possible because of the systematic large scale dynamic structure (the cosmic expansion) where $t(\operatorname{cosm})$ would be the proper time of a particle which flows with the stream. One could speak of a beginning of physical time only if there has indeed been an initial singularity (the big bang). That wouid have to be understood in the sense that before a certain hypothetical event, i.e. the big bang, we could not 
construct in our mind anything of physical significance but we would still ask what happened before. In other words the absence of physical events would preclude also a time measurement even though we can still extend our mathematical scale further backwards in time. But this would be a purely arbitrary, fruitless and unnecessary play of the mind.

Time is both, local and macroscopic. It is local because of the interdependence of time and distance measurements in the relativistic sense, and it is macroscopic because time is an emergent aspect which gains its meaning only in the macro world. One of the reasons for that claim (and we shall see more about it later) is the idea in quantum mechanics that an isolated system in its fundamental state has no cause for change, it experiences no time, i.e., in the state equations there is no time parameter left. A different case for timeless existence would be the photon which may be on its way for billions of years, yet in its reference system emission and absorption are simultaneous. In this case we find agreement with the result obtained from the first idea, that it experiences no change while on its way. We have disregarded the cosmological doppler shift which does reduce its relative energy. We disregard this because we consider it a metric phenomenon, not a physical effect. However, to the extent that the photons are not perfectly isolated from the universe, we would have to expect a small time dependency of its state parameters, i.e., its energy would become a function of time. This physical, as opposed to the metric, explanation of the redshift is a possibility which present doctrine (Kuhn's paradigm) does not favor because it does not fit well with the rest of our present experience. But its definite possibility must be kept in mind in order to realize that most of the present work in cosmology depends on the current interpretation of the redshift.

A number of different theories on time have been advanced in attempts to reduce time to other, supposedly more fundamental, concepts. Examples are the causal theory, thermodynamic theory, statistical theory, cosmological theory (Gold), etc. But we find that in principle these attempts are misguided and indeed, none of these is consistent because a time concept is necessary prior to any description of nature, i.e. time is a primitive concept. It being a primitive concept also means that it is by necessity uni-directional, i.e., any discussion of reversibility can only be concerned with reversibility of particular processes or laws. It is now interesting to note that all strict determinism would imply a general reversibility. All classical laws of mechanics and electrodynamics fall in this group. Thermodynamics, however, does not. Already the beat conduction equation is not time-symmetric. We come to suspect, therefore, that irreversibility may be an emerging property of systems and that the time of the classical laws is the time of automatons, i.e., something which does not really concern the real world because the classical laws are only a very insufficient approximation (or better, model) of reality. See, however, [1] for the arguments by Boltzmann who suspected that the direction of time would be a function of location in space!

The definition which refers to "equal processes" entails a basic feature of all realizations based upon it. Every cycle of whatever process we select can only be realized with a small but finite error. That means that there is no perfect clock. In the best case the noise introduced in the time keeping process will be uncorrelated in the measurement of the basic time intervals, i.e., it will lead to a random walk in the time indicated. Normally, however, clocks (or natural processes serving as clocks) will suffer in addition also systematic changes in rate. We say that this kind of noise is correlated in time, and because it is persistent over some time it will lead to substantial clock errors, much larger than the intrinsic random walk. There are three ways to combat these clock deficiencies: Shield the clock from external disturbances (and by proper design avoid internal relaxation phenomena), select a better process as the basic clock process (a process with a greater intrinsic stability such as narrower spectral lines as frequency reference in atomic clocks), or average over many independently operating clocks. This last choice is often a council of despair if nothing else can be done but it offers the additional great benefit of increasing the reliability of operations. Time keeping and statistics are, therefore, intimately connected. And from a higher point of view one can even say that this connection is in another sense a fundamental one. The most basic and most general process we can find in nature is the decay of order, the increase in entropy in every closed system. This would lead to the concept of a statistical clock such as a design where we would count seconds after the same (decreasing over long times with the loss of material as given by the half life time) number of 
decaying radium atoms have been measured. At this time, this would not be competitive with current atomic clocks but I mention it to illustrate the principle.

In striking contrast to the "static" aspect of time in classical physics where experiments are assumed to be exactly repeatable independent of the time, time assumes an altogether different role in biology and more rerentlv, in cosmology. Evolution is another aspect of irreversibility and time in this context assumes the scuse of Becoming as opposed to the classical Being; existence according to "laws" which are valid for all times and for all places. Exactly the same double aspect, Being and Becoming, appears also in History and in all sciences that are concerned with systems as opposed to the fundamental phenomena. In Being the only relation between events is the earlier - later (or simultaneous) whereas in Becoming, in addition to this relation we also have to specify "when".

Relativistic physics leads to two very striking conclusions: First, the space-time picture of the world assigns to time a static role. Things do not happen, they are, in this frozen four dimensional world. Observing minds only become aware of successive three dimensional cross sections as they move along the worldline. This suggests a concept of the world very much along the lines of Parmenides' ideas. What is is unchanging. Change is only a feature of the world as it appears to the mind (the servant of the Self which, because of its desires creates change; and change is what it wants).

The second conclusion corroborates this. The way we split the world into space and time depends entirely upon the subjective choice of a reference system. Therefore, the flow of time as such can't be part of a purely objective world, it must be subjective. This is an additional reason why we should assign a purely ideal character to time. It is a nocessary category of cognition, as first pointed out by Kant, which is, however, based upon a real feature of the world which we do not understand and which must be part of the ultimate reality. The measures of time which we find useful are, however, entirely conventional and solely depend upon how we define identical processes. We see that in that definition is also contained the problem of whether or not we should admit the measurement of principally different physical times.

The answer is that we should not; that the introduction of two or several intrinsically different times, such as e.g. atomic time; gravitational time; statistical time; etc.; that if indeed the need for such conceptions would arise, that in that case our physical understanding would be deficient. In other words, if we should find (no such evidence has been found yet within the very small errors of measurement of less than $1 \mathrm{E}^{-12}$ per year) that a time scale used for the description of dynamical phenomena, i.e. orbits of celestial bodies, would deviate systematically from atomic time, then our theories of gravitation will have to take that into account. An attempt to bury such "cosmological" effects in the introduction of different time scales would be misguided because it would defeat the very purpose of the time parameter, i.e., to serve as common basis for the description of all processes, throughout the universe.

We can conclude at this point that as a theoretical basis we must decide on the standard process; in practice on the best clock, where best means that which is in best agreement with the consensus of other time keepers. But it also can be said that it would be foolish, or at least unnecessarily wasteful and confusing, if we would bother the public with a variety of different times, all disseminated, just because we feel that the right tool should be chosen for the right job. That would follow the bad example of some other professions who try to force everybody to learn their trade by making ordinary life affairs and things so complicated that only experts can hope to understand. Examples come readily to mind: that is why we need tax advisors! On the other hand, it is perfectly useful and practical for the expert to use specialized tools for special purposes. This is where the many special "times" in astronomy come in: We do not disseminate local sidereal time, or ephemeris time, or TDB (to be discussed below), but we certainly find them useful for our computations. If we desire consistency, then we should use but a single, publicly available time, UTC (or possibly TAI) as arguments in tables or reports and leave the finer distinctions and conversions to the expert. 


\section{THE INTERNATIONAL SYSTEM OF UNITS SI}

The system of measurement units that we have accepted is the SI which is also in its ideal principles a system for local use, the same way as the concept of time itself. This locality has been specifically recognized in the case of the time measures, albeit only gradually. At the time of the adoption of the present SI Second in 1967, it was implied that the definition had to be in the concept of proper time. Only much later, when the exast meaning of TAI had to be considered, was the distinction between local (proper) time and coordinate time included in the actual wording of a recommendation. Regarding the other base units of the SI, the evolution of the system is not yet completed. The Second has become the most important unit: by having defined the speed of light once and for all, the unit of length is now also besed upon the Second. In addition, the Second is based not on a prototype, but on a postulated constant of nature - the energy levels of the cesium atom. Efforts are under way to continue in this direction: away from prototypes towards constants of nature.

In practical terrestrial timing applications, the need to reduce remote time measurements relativistically became very obvious with the first satellite timing experiments. However, it took some time to make this part of relativity a part of the timing engineer's tool box (see [2] through [12] for a discussion of details). As an official act, the CCDS has at first clarified the principles involved, and has defined in 1970 the International Atomic Time (TAI) as a coordinate (and coordinated) time [13]. The wording (translated from the French) is:

"The TAI is the temporal reference coordinate established by the Bureau International de l'Heure (BIH) on the basis of the readings of atomic clocks that operate in various establishments in conformance with the definition of the Second, the unit of time of the International System of Units (SI)." (Recommendation S 2, 1970).

This definition of TAI has been augmented with a declaration in 1980 that clarified the meaning in the relativistic context. This became necessary because the above wording only implies the reference time TAI to be coordinated because TAI is defined on the basis of the clock readings in the contributing establishments. The unit of time, as it is defined as a base unit of the SI, however, necessarily refers to proper time. Wherever we operate a Cesium frequency standard, it gives us the time measure as a proper unit, even though this also was not explicitly spelled out in the original definition. This CCDS declaration is as translated [13]:

"TAI is a coordinate time scale, defined in a geocentric reference frame with the SI Second as scale unit as it is realized on the rotating geoid. Therefore, it can be extended to a fixed or moving point in the vicinity of the Earth with sufficient accuracy at the present state of the art by the application of the first order corrections of the General Theory of Relativity; i.e., the corrections for the differences in the gravitational potential and the differences of speed, in addition to the rotation of the Earth."

The CCIR Study Group VII has issued a report [14] that deals in some detail with the cases of a portable clock near the surface of the Earth, and with electromagnetic signals used for remote synchronization. The report is in essential agreement with the CCDS documents with some additional detail of importance for terrestrial applications.

\section{THE PRESENT SITUATION IN ASTRONOMY REGARDING STANDARDS OF TIME}

The old Ephemeris Time as it was used as the argument for orbital computations, was replaced in 1977 by Dynamical Time, which included relativity considerations with scaling (changing the rate of the clocks to compensate for the gravitational potential at the point of origin for the purpose of avoiding a secular 
runoff; see [15]). Two main guiding principles were used in this replacement. First, the moon was to be replaced with the more accurate cesium standard (even though there was ambiguity in the wording!). And second, continuity with the past ET was considered essential. However, the new space applications of precise time, particularly in pulsar research, suggest further evolution. This is very significant because by far the most stringent requirements for long-term clock stability, and accuracy in the relativity corrections that must be applied in the reduction of observations, come from astrodynamics and astronomical research. Guinot and Seidelmann [16] discuss the histery and propose further developments.

The 1977 decisions regarding Dynamical Time were to some degree premature and, therefore, unfortunate. The name was entirely confusing but even in the text, an unfortunate ambiguity existed in respect to the choice of the scale unit: was it defined at the epoch or for all time? In addition, no agreement could be reached about the role of TAI: was it, as the CCDS later spelled out, a coordinate time, or in astronomical context, a proper time? And last but not least: the scaling! At the time, we obviously did not see clearly enough the importance of consistency in our system of measurement. In fact by scaling we gain nothing if -- and that is a crucial point - we do not disseminate the time. In that case, we do not have to worry about runoff! On the other hand, dropping the scaling allows us to use our physical system of measurement consistently.

IAU Colloquium 127 has now provided a long overdue clarification. Relativity has been included in all concepts from the beginning. Recommendation G2 [17] specifically mentions two coordinate systems, the (solar) barycentric and a geocentric system; it specifies that the SI (i.e., the second, the meter, c, etc.) should be extended to outer space without scaling factors; and it links the time coordinates to atomic clocks that operate in conformance with the definition of the Second.

Recommendations $\mathrm{T} 1$ and $\mathrm{T} 2$ establish a consistent nomenclature for the various time-like arguments that need to be distinguished as a consequence of the above recommendations. This nomenclature takes into account the previously intioduced time-ilke arguments that are used in the ephemerides: Terrestrial Dynamical Time TDT, and Barycentric Dynamical Time TDB, the former originally taken as TAI + $32.184 \mathrm{~s}$, and usually considered as the relativistic successor to ET. TDB is reckoned at the SI rate with scaling (which effectively assigns a number to the Cesium standard frequency that is different from the SI). $T 1$ and $T 2$ also introduce new arguments in conformance with $G 1$ and $G 2$ : Geocentric Coordinate Time TCG, and Barycentric Coordinate Time TCB. And lastly, it also introduces an ideal Terrestrial Time TT that is practically TAI but without the very small errors of implementation.

First of all, it must be noted that the French names determine the abbreviations. And even though this seems like a bewildering profusion of different time scales, the method is clearer by grouping them in the following way (see also Table 1):

Dynamical times were conceived for the sole purpose of providing relativistic successors to the ET. This historical origin, together with the urgency with which they were introduced before a systematic position could be reached, as it exists now, together with the 32.184 second offset inherited from ET, explains why better distinctions and definitions were needed. Moreover, the name Dynamical is misleading because it refers to the intended use, while the scale is really an atomic time. The scaling of the rate means that the standard units (e.g., of mass) must also be scaled if they are connected with TDB, a serious complication that is avoided with the introduction of the "Coordinate" times. These are not scaled but adopt the SI at the origins. This is in the spirit of the SI, which is tacitly assumed to be a proper system of units. Another problem we face is that we need to be able to conceptually separate TAI as an established, operational time, from its ideal concept, which can be better approximated after the fact by reprocessing and the inclusion of additional information (possibly, pulsar observations). That is the reasoning behind TT. It is practically identical with TDT except that the separation of the realized from the ideal time was not explicitly included in exactly this sense in the definition of TDT, which was rather vague on this point [15]. In addition, the possibly misleading implications arising from the name Dynamical are now avoided 
with TT. The only blemish in this otherwise logical scheme is the continuation of the 32.184 second offset on the right side of the scheme. This is confusing and a potential source of error.

Lastly we must also recognize that TAI is a coordinate time that is not in conformance with G1 and G2 because it is not a "centric" time, but is defined on the geoid. However, all our observations are necessarily referred to it and we must, therefore, establish a connection with it. This is the purpose of transforming to TCG.

It is hoped that this nomenclature will allow unambiguous references in the discussion of work that deals with precision space observations, even though not all of these scales will actually come to be used. The planetary ephemeris tapes that are presently computed in reference to TDB, but published in reference to TDT, will probably continue to use TDB.

TABLE 1

\title{
RELATIONS BETWEEN ASTRONOMICAL TIMESCALES
}

1976 Recommendations

1990 Proposals

TDT

(on the Geoid)

identical with

TT

Successor to ET

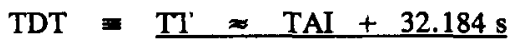

Geocentric Coordinate Time TCG

TCG - TT $\approx 6.969291 \cdot 10^{-10} \cdot \mathrm{VT}$

| --- four-dimensional transformation to the barycenter --- |

secular term $\approx 1.480813 \cdot 10^{-8} \cdot \mathrm{VT}$

TDB

\author{
$\mathrm{TCB}-\mathrm{TDB} \approx 1.550505 \cdot 10^{-8} \cdot \mathrm{VT}$ \\ $\mathrm{VT}=$ (date in days -1977 January 1, Oh) $\cdot 86400 \mathrm{~s}$ \\ determined in TAI
}




\section{NOTES AND LITERATURE:}

[1] Ludwig Boltzmann (1912) Vorlesungen ueber Gastheorie. II. Teil. Johannn Ambrosius Barth, Leipzig. See p. 257 (Paragraph 90): B. argues that in a closed universe there must eventually be as many regions where entropy increases as regions where it decreases. "Therefore" there is no preferred direction of time, as there is no up or down in space. Since this was written long hefore relativity theory (1912 is the date of a second edition) it is remarkable that B. clearly states a senecpt of time which is radically different from Newton's. However, the reasoning is not correct because it tacitly presupposes a time of reference against which one can state whether anything increases or decreases. The reasoning assumes tacitly what it tries to disregard: that time is a primitive concept.

[2] Louis Marton (ed.) (1977) Advances in Electronics and Electron Physics. Chapter 2. Academic Press NY. ISBN 0-12-014644-4 LCC\# 49-7504 Gives an overview of time measurement with many references.

[3] C. O. Alley, "Introduction to Some Fundamental Concepts of General Relativity and to Their Required Use in Some Modern Timekeeping Systems," Proceedings of the Thirteenth Annual Precise Time and Time Interval (PTTI) Applications and Planning Meeting, pp. 687-727, 1981 (NASA Conference Publication 2220).

[4] J. C. Hafele and R. E. Keating, "Around the World Atomic Clocks," Science, vol. 177, pp. 166-170, 14 July 1972.

[5] C. O. Alley, "Relativity and Clocks," Proceedings of the 33rd Annual Symposium on Frequency Control, pp. 4-39A, 1979.

[6] I. I. Shapiro, "Down to Earth Relativity," Proceedings of the Teuth Annual Precise Time and Time Interval (PTT) Applications and Planning Meeting, pp. 549-568, 1978 (NASA Technical Memorandum 80250).

[7] N. Ashby and D. W. Allan, "Practical Implications of Relativity for a Global Coordinate Time Scale," Radio Science, vol. 14, pp. 649-669, 1979. This is a particularly important paper for timing applications: it discusses many specific examples, from clock trips to satellite time comparisons.

[8] N. Ashby and D. W. Allan, "Coordinate Time on and near the Earth," Physical Review Letters, vol. 53, p. 1858, 1984.

[9] C. O. Alley, et al., "Differential Comparison of the One-Way Speed of Light in the East-West and West-East Directions on the Rotating Earth, "Proceedings of the Twentieth Annual Precise Time and Time Interval (PTTI) Applications and Planning Meeting, pp. 261-286, 1988 (available from the U.S. Naval Observatory). For full details of this interesting experiment see [19].

[10] D. W. Allan and N. Ashby, "Coordinate Time in the Vicinity of the Earth," in International Astronomical Union Symposium No. 114: Relativity in Celestial Mechanics and Astrometry, J. Kovalevsky and V. A. Brumberg, Eds., pp. 299-313, Dordrecht, Holland: D. Reidel Pub. Co., 1986.

[11] D. W. Allan, M. A. Weiss, and N. Ashby, "Around-the-World Relativistic Sagnac Experiment," Science, vol. 228, pp. 69-70, 5 April 1985.

[12] J. B. Thomas, "A Relativistic Analysis of Clock Synchronization. "Proceedings of the Sixth Annual Precise Time and Time Interval (PTT) Applications and Planning Meeting pp. 425-439, 1974 (available from the U.S. Naval Observatory). 
[13] Comité Consultatif pour la Définition de la Seconde, "Declaration et Note", 9e Session, Bureau International des Poids et Mesures (BIPM), Pavillon de Breteuil, F-92310 Sèvres, France, 1980.

[14] Recommendations and Reports of the CCIR, "Relativistic Effects in a Terrestrial Coordinate Time System", ITU Geneva, Report 439-4, Volume VII, pp. 134-138, 1986. A slightly modified version will be published in the "Green Book" of the XVIIth Plenary Assembly, Düsseldorf, Germany, 1990.

[15] G.M.R. Winkler and T. van Flandern, "Ephemeris Time, Relativity, and the Problem of Uniform Time in Astronomy", Astron. J. 82, pp. 84-92, January 1977.

[16] B. Guinot and P. K. Seidelmann, "Time Scales: their history, definition and interpretation," Astron. Astrophys. vol. 194, pp. 304-308, 1988.

[17] In I.A.U. Colloquium 127 Reference Systems, Virginia Beach, Virginia, USA, 14-20 October 1990. Eds. J. A. Hughes, G. Kaplan, and C. Smith, U.S. Naval Observatory 1991. 